\title{
A FUNCTORIAL APPROACH TO WEAK AMENABILITY FOR COMMUTATIVE BANACH ALGEBRAS
}

\author{
by VOLKER RUNDE
}

(Received 1 March, 1991)

1. Introduction. Let $A$ be a commutative algebra, and let $M$ be a bimodule over $A$. A derivation from $A$ into $M$ is a linear mapping $D: A \rightarrow M$ that satisfies

$$
D(a b)=a D b+(D a) b \quad(a, b \in A) .
$$

If $M$ is only a left $A$-module, by a derivation from $A$ into $M$ we mean a linear mapping $D: A \rightarrow M$ such that

$$
D(a b)=a D b+b D a \quad(a, b \in A) .
$$

Each $A$-bimodule $M$ is trivially a left module. However, unless it is commutative, i.e.

$$
a x=x a \quad(a \in A, x \in M),
$$

the two classes of linear operators from $A$ into $M$ characterized by (1) and (2), respectively, need not coincide.

In [1] Bade, Curtis, and Dales coined the name weakly amenable Banach algebra for a commutative Banach algebra $A$ such that there is no non-zero continuous derivation from $A$ into a commutative Banach $A$-bimodule, or equivalently, to speak in terms of Hochschild cohomology, $\mathscr{H}^{1}(A, M)=\{0\}$ for each commutative Banach $A$-bimodule $M$. As indicated by the adverb weakly, every commutative, amenable Banach algebra $A$ is weakly amenable [2, Proposition 43.14], whereas there are commutative Banach algebras which are weakly amenable, but not amenable [1]. Each left module $M$ over a commutative Banach algebra $A$ is naturally turned into a commutative $A$-bimodule via

$$
x a:=a x \quad(a \in A, x \in M) .
$$

Hence, we may define the class of weakly amenable Banach algebras in terms of left Banach modules.

Definition 1.1. Let $A$ be a commutative Banach algebra. If there is no non-zero continuous derivation from $A$ into a left Banach $A$-module, $A$ is called weakly amenable.

The present paper provides a functorial approach to weak amenability. We shall assign to each commutative Banach algebra $A$ a left Banach $A$-module $\Omega(A)$, the Banach module of differentials over $A$, which is characterized by a universal property such that $\Omega(A)=\{0\}$ if and only if $A$ is weakly amenable. The construction of $\Omega(A)$ will yield an alternative proof of a result due to Grønbak, which characterizes the weak amenability of $A$ in terms of the projective tensor product $A \otimes A$. Further, we shall investigate functorial properties of the assignment $A \mapsto \Omega(A)$. Modules of differentials are well known in commutative algebra (see [9]); we shall transfer some of the results known to hold for modules of differentials in the purely algebraic theory to the Banach algebra situation.

I am grateful to Dr. Jörg Eschmeier at Münster and to Professor H. G. Dales at Leeds who made valuable comments and suggestions. 
2. Banach modules of differentials. Let $A$ be a commutative algebra with identity over a commutative unital ring $R$, and consider a category whose objects are pairs $\left(d_{M}, M\right)$, where $M$ is a left $A$-module and $d_{M}: A \rightarrow M$ is a derivation. Given two such pairs $\left(d_{M}, M\right)$ and $\left(d_{N}, N\right)$, a morphism $\left(d_{M}, M\right) \rightarrow\left(d_{N}, N\right)$ is a homomorphism $h: M \rightarrow N$ of $A$-modules such that $d_{N}=h \circ d_{M}$. Recall that an object $C_{0}$ of a category is called universally repelling if for every other object $C$ of this category there is a unique morphism $C_{0} \rightarrow C$; universally repelling objects are unique up to canonical isomorphism. It is a well known result from commutative algebra (see [9, Chapter 10.26]) that the category considered above has universally repelling objects. The module belonging to such a universal pair is called the module of (Kähler) differentials over $\boldsymbol{A}$.

In this section, we shall give an analogue of the result sketched above in the Banach algebra setting. Before we state this theorem in detail and start proving it, let us fix a very simple but useful observation as a lemma:

Lemma 2.1. Let $A$ be a commutative algebra, let $M$ be a left $A$-module, and let $D: A \rightarrow M$ be a derivation. Then $D e=0$ for every idempotent $e \in A$.

If $A$ is a non-unital commutative Banach algebra, then all left Banach $A$-modules are Banach $A^{\#}$-modules in a canonical way, where $A^{\#}$ is the Banach algebra we obtain by adjoining an identity to $A$. By Lemma 2.1 , all derivations from $A$ extend canonically to $A^{*}$. Therefore, we can avoid the restriction to unital Banach algebras.

THEOREM 2.2. Let $A$ be a commutative Banach algebra. Then there is a left Banach $A$-module $\Omega(A)$ and a continuous derivation $d_{A}: A \rightarrow \Omega(A)$ with the following universal property.

For each continuous derivation $D: A \rightarrow M$ into a left Banach $A$-module $M$, there is a unique continuous homomorphism $h_{D}: \Omega(A) \rightarrow M$ such that $D=h_{D}{ }^{\circ} d_{A}$, i.e. the diagram

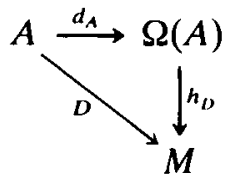

is commutative. $\Omega(A)$ is unique up to topological isomorphism.

Proof. Without loss of generality assume $A$ to have an identity element 1 . Let $A \otimes A$ denote the projective tensor product, and consider the diagonal homomorphism $\delta: A \otimes A \rightarrow A$ which is given by

$$
\delta(a \otimes b)=a b \quad(a, b \in A) .
$$

Put $I_{\Delta}:=\operatorname{ker} \delta$. Let $x \in I_{\Delta}$; by [2, Proposition 42.12] there are sequences $\left(a_{k}\right)$ and $\left(b_{k}\right)$ in $A$ such that

$$
x=\sum_{k=1}^{\infty} a_{k} \otimes b_{k} \quad \text { and } \quad \sum_{k=1}^{\infty} a_{k} b_{k}=0
$$

This means that

$$
\begin{aligned}
x & =\sum_{k=1}^{\infty} a_{k} \otimes b_{k}-\sum_{k=1}^{\infty} a_{k} b_{k} \otimes 1 \\
& =\sum_{k=1}^{\infty} a_{k}\left(1 \otimes b_{k}-b_{k} \otimes 1\right) .
\end{aligned}
$$


Hence, $I_{\Delta}$ is the Banach $A$-submodule of $A \otimes A$ which is generated by the elements

$$
1 \otimes a-a \otimes 1 \quad(a \in A) .
$$

Define $d: A \rightarrow I_{\Delta}$ by

$$
d a=1 \otimes a-a \otimes 1 .
$$

A quick computation shows that

$$
d(a b)-a d b-b d a=(d a)(d b) \in I_{\Delta}^{2} \quad(a, b \in A) .
$$

Put $\Omega(A):=I_{\Delta} / \overline{I_{\Delta}^{2}}$. Then $d_{A}: A \rightarrow \Omega(A)$, defined by

$$
d_{A} a=1 \otimes a-a \otimes 1+\overline{I_{\Delta}^{2}} \quad(a \in A)
$$

is a continuous derivation. Now, let $M$ be an arbitrary left Banach $A$-module, and let $D: A \rightarrow M$ be a continuous derivation. Define a continuous $A$-module homomorphism $H_{D}: A \otimes A \rightarrow M$ via

$$
H_{D}(a \otimes b)=a D b \quad(a, b \in A) .
$$

It is easy to see that

$$
H_{D}((d a)(d b))=0 \quad(a, b \in A) .
$$

Since $I_{\Delta}$ is generated by the set $\{d a \mid a \in A\}$, this means that $H_{D}$ vanishes on $\overline{I_{\Delta}^{2}}$. Thus $h_{D}: \Omega(A) \rightarrow M$, where

$$
h_{D}\left(x+\overline{I_{\Delta}^{2}}\right)=H_{D}(x) \quad(x \in \Omega(A))
$$

is a well defined, continuous homomorphism of $A$-modules. From the construction,

$$
D=h_{D} \circ d_{A}
$$

is obvious. Since $\Omega(A)$ is the closed $A$-linear span of $d_{A}(A)$, it follows from (3) that $h_{D}$ is uniquely determined by that property. To prove the uniqueness of $\Omega(A)$ up to topological isomorphism is pure arrow theory.

We call $\Omega(A)$ the Banach module of differentials over $A$. Note that for a non-unital commutative Banach algebra $A, \Omega(A)=\Omega\left(A^{\#}\right)$. If $A$ has an identity element $1, \Omega(A)$ is unit-linked, i.e.

$$
1 x=x \quad(x \in \Omega(A)) .
$$

It is an immediate consequence of the universal property of $\Omega(A)$ that $A$ is weakly amenable if and only if $\Omega(A)=\{0\}$. In other words we have the following result.

COROLlaRY 2.3. Let $A$ be a commutative Banach algebra. Then $A$ is weakly amenable if and only if $I_{\Delta}^{2}$ is dense in $I_{\Delta}$.

A different approach to this intrinsic characterization of weakly amenable Banach algebras has been given by Grønbæk in [6]. There is an analogous statement for amenable, not necessarily commutative Banach algebras ([7, Theorem VII.2.20] or [3, Theorem 1.3]), which characterizes amenable Banach algebras in terms of the diagonal ideal $I_{\Delta}$ (in the non-commutative case the definition of $I_{\Delta}$ differs slightly from the one in the proof of Theorem 2.2). For a commutative Banach algebra $A$ this result asserts that $A$ is amenable, if and only if both $A$ and $I_{\Delta}$ have bounded approximate identities. 
Immediately from the construction of $\Omega(A)$, we obtain as well:

COROLlaRY 2.4. Let $A$ be a commutative Banach algebra which is generated by $\left\{a_{\lambda} \mid \lambda \in \Lambda\right\}$. Then $\Omega(A)$ is generated by $\left\{d a_{\lambda} \mid \lambda \in \Lambda\right\}$.

In contrast to the purely algebraic theory of differentials (see [9, (26.E) Example 1]), if $A$ is a Banach algebra generated by a family $\left\{a_{\lambda} \mid \lambda \in \Lambda\right\}$ of elements which are algebraically independent over $\mathbb{C}, \Omega(A)$ need not be the free $A$-module generated by $\left\{d a_{\lambda} \mid \lambda \in \Lambda\right\}$ : For example, $\mathscr{C}[0,1]$ is generated by the element

$$
x:[0,1] \rightarrow \mathbb{C} \quad t \mapsto t,
$$

which is clearly transcendental over $\mathbb{C}$. Since $\mathscr{C}[0,1]$ is weakly amenable by $[2$, Theorem 43.12], or by [1, Theorem 1.4], $\Omega(A)=\{0\}$. It may also occur that a commutative Banach algebra $A$ is generated by a single transcendental element $a \in A$ such that $d_{A} a \neq 0$, but $\Omega(A)$ is not the free module generated by $d_{A} a$. For

$$
\mathbb{D}=\{\zeta \in \mathbb{C}|| \zeta \mid<1\}
$$

let $A(\mathbb{D})$ be the Banach algebra of those continuous functions on $\overline{\mathbb{D}}$ which are analytic on $\mathbb{D}$ itself. $A(\mathbb{D})$ is generated by

$$
z: \mathbb{D} \rightarrow \mathbb{C} \quad \zeta \mapsto \zeta .
$$

Since $A(\mathbb{D}) \ni f \mapsto f^{\prime}(0)$ is a non-zero continuous derivation, $d_{A(\mathbb{D})} z$ cannot be zero. On the other hand, if $d_{A(\mathbb{D})} z$ were a basis for $\Omega(A(\mathbb{D}))$, there would be an isomorphism $h: \Omega(A(\mathbb{D})) \rightarrow A(\mathbb{D})$ of $A(\mathbb{D})$-modules. Define $D=h \circ d_{A(\mathbb{D})}$. Then $D: A(\mathbb{D}) \rightarrow A(\mathbb{D})$ is a derivation. Since $A(\mathbb{D})$ is semisimple, $D=0$ by [2, Theorem 18.21]. This is a contradiction.

3. Functorial properties of $\Omega$. Let $A$ and $B$ be commutative Banach algebras, and let $\phi: A \rightarrow B$ be a continuous homomorphism. Then $\Omega(B)$ is a left Banach $A$-module in a natural way, and $d_{B} \circ \phi$ is a continuous derivation from $A$ into $\Omega(B)$. By Theorem 2.2, there is a unqiue continuous homomorphism $\Omega(\phi): \Omega(A) \rightarrow \Omega(B)$ which makes the diagram

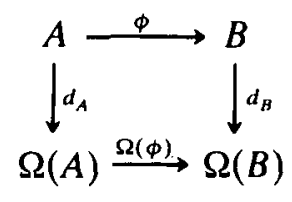

commutative. If $C$ is a third commutative Banach algebra, and $\psi: B \rightarrow C$ is a continuous homomorphism, it is easy to see that $\Omega(\psi \circ \phi)=\Omega(\psi) \circ \Omega(\phi)$, i.e. $\Omega$ is a covariant functor.

The observation that $\Omega$-in the context of analytical local algebras-has functorial properties can already be found in [4]. However, there seem to be no subsequent investigations in this direction.

Whenever we encounter a functor, certain questions arise naturally. What does it do to complexes? Does it do harm to exact sequences? In our situation the following question is of particular interest: Let $A$ be a commutative Banach algebra, and let $I \subset A$ 
be a closed ideal: What happens to the canonical exact sequence

$$
0 \rightarrow I \stackrel{\iota}{\rightarrow} A \stackrel{\pi}{\rightarrow} A / I \rightarrow 0
$$

when $\Omega$ is applied to it?

Evidently, $\Omega(\phi)=0$ if $\phi=0$. Thus, the answer to the question, what happens to a complex, when we apply $\Omega$ to it, is straightforward.

Proposition 3.1. Let $\left\{A_{k} \mid k \in \mathbb{Z}\right\}$ be a family of commutative Banach algebras, and let $\phi_{k}: A_{k} \rightarrow A_{k+1}(k \in \mathbb{Z})$ be continuous homomorphisms such that

$$
\cdots \rightarrow A_{k} \stackrel{\phi_{k}}{\longrightarrow} A_{k+1} \rightarrow \cdots
$$

is a complex. Then

$$
\cdots \rightarrow \Omega\left(A_{k}\right) \stackrel{\Omega\left(\phi_{k}\right)}{\longrightarrow} \Omega\left(A_{k+1}\right) \rightarrow \cdots
$$

is a complex of Banach spaces.

Let $A$ and $B$ be commutative Banach algebras, and let $\phi: A \rightarrow B$ be a continuous homomorphism. We want to see now what properties of $\phi$-injectivity, surjectivity, a dense range-are inherited by $\Omega(\phi)$.

Proposition 3.2. Let $A$ and $B$ be commutative Banach algebras, and let $\phi: A \rightarrow B$ be a continuous homomorphism with dense range. Then the range of $\Omega(\phi)$ is dense in $\Omega(B)$.

Proof. Since $\phi(A)$ is dense in $B$, an application of Corollary 2.4 yields that the $B$-module generated algebraically by $d_{B}(\phi(A))$ is dense in $\Omega(B)$. Again by the density of $\phi(A)$ in $B$, the same holds for the $\phi(A)$-module generated by $d_{B}(\phi(A))$, i.e. for the range of $\Omega(\phi)$.

A trivial consequence of Proposition 3.2 is that weak amenability is inherited via morphisms with dense range: this merely qualitative assertion can be proved directly in a few lines; a similar statement holds for amenability [8, Proposition 5.3].

Lemma 3.3. Let $A$ and $B$ be commutative Banach algebras, and let $\phi: A \rightarrow B$ be $a$ continuous homomorphism. Then $\Omega(A) / \operatorname{ker} \Omega(\phi)$ becomes a left Banach $A / \operatorname{ker} \phi$-module via

$$
(a+\operatorname{ker} \phi)(x+\operatorname{ker} \Omega(\phi)):=a x+\operatorname{ker} \Omega(\phi) \quad(a \in A, x \in \Omega(A)) .
$$

Proof. To see that the module multiplication is well defined we have to show that

$$
a x \in \operatorname{ker} \Omega(\phi) \quad(a \in \operatorname{ker} \phi, x \in \Omega(A)) .
$$

Let $a \in A$, and $x \in \Omega(A)$. Since $\Omega(A)$ is generated by $d_{A}(A)$, we may assume that $x=d_{A} y$ for some $y \in A$. Then

$$
a x=a d_{A} y=d_{A}(a y)-y d_{A} a .
$$

As $\operatorname{ker} \phi$ is an ideal, we have $a y \in \operatorname{ker} \phi$, and hence, $d_{A}(a y), d_{A} a \in \operatorname{ker} \Omega(\phi)$. This implies $a x \in \operatorname{ker} \Omega(\phi)$. 
Proposition 3.4. Let $A$ and $B$ be commutative Banach algebras, and let $\phi: A \rightarrow B$ be a continuous homomorphism such that

$$
A^{\Phi} B \rightarrow 0
$$

is exact. Then

$$
\Omega(A) \stackrel{\Omega(\phi)}{\longrightarrow} \Omega(B) \rightarrow 0
$$

is an exact sequence of left Banach A-modules.

Proof. There is a natural isomorphism $\hat{\phi}: A / \operatorname{ker} \phi \rightarrow B$. By the preceding lemma, $\Omega(A) / \operatorname{ker} \Omega(\phi)$ is a left Banach $A / \operatorname{ker} \phi$-module and hence a $B$-module. Define $\hat{d}_{A}: A / \operatorname{ker} \phi \rightarrow \Omega(A) / \operatorname{ker} \Omega(\phi)$ by

$$
\hat{d}_{A}(a+\operatorname{ker} \phi)=d_{A} a+\operatorname{ker} \Omega(\phi) \quad(a \in A) .
$$

Since $d_{A}(\operatorname{ker} \phi) \subset \operatorname{ker} \Omega(\phi), \hat{d}_{A}$ is well defined and clearly a derivation. Thus $\hat{d}_{A}{ }^{\circ} \hat{\phi}^{-1}$ is a derivation from $B$ into the left Banach $B$-module $\Omega(A) / \operatorname{ker} \Omega(\phi)$. It follows from Theorem 2.2 that there is a continuous $B$-module homomorphism $h: \Omega(B) \rightarrow$ $\Omega(A) / \operatorname{ker} \Omega(\phi)$ with

$$
\hat{d}_{A} \circ \hat{\phi}^{-1}=h \circ d_{B}
$$

Define

$$
\widehat{\Omega(\phi)}: \Omega(A) / \operatorname{ker} \Omega(\phi) \rightarrow \Omega(B) \text { by } \widehat{\Omega(\phi)}(x+\operatorname{ker} \Omega(\phi))=\Omega(\phi) x .
$$

Now, (4) implies that

$$
(\widehat{\Omega(\phi)} \circ h)\left(d_{B} b\right)=\left(\widehat{\Omega(\phi)} \circ \hat{d}_{A} \circ \hat{\phi}^{-1}\right)(b)=d_{B} b \quad(b \in B) .
$$

Since $\Omega(B)$ is generated by $d_{B}(B)$, this means that $h$ is a right inverse of $\widehat{\Omega(\phi)}$. Therefore, $\widehat{\Omega(\phi)}$ is an isomorphism, and $\Omega(\phi)$ is onto.

In contrast, when $\Omega$ is applied to an exact sequence of commutative Banach algebras

$$
0 \rightarrow A \stackrel{\oplus}{\rightarrow} B,
$$

the situation is much less satisfactory. Let $A=\mathscr{C}^{1}[0,1]$, the Banach algebra of all continuously differentiable functions on $[0,1], B=\mathscr{b}[0,1]$, and $\phi=\iota$, the natural embedding, or $A=A(\mathbb{D}), B=\mathscr{C}(\mathbb{D})$, and again $\phi=\imath$. In both cases $\Omega(B)=\{0\}$. Hence, $\Omega(\phi)$ must be zero, whereas $\Omega(A)$ is evidently not trivial. Note that in these two examples, $\phi$ is the Gelfand homomorphism-with dense range in the first case, as isometry in the second.

Before we state the main result of this section, we want to make clear what we mean by a topologically exact sequence.

Definition 3.5. Let $\left\{X_{k} \mid k \in \mathbb{Z}\right\}$ be a family of Banach spaces, and let $T_{k}: X_{k} \rightarrow X_{k+1}$ $(k \in \mathbb{Z})$ be linear operators such that

$$
\overline{T X_{k}}=\operatorname{ker} T_{k+1} \quad(k \in \mathbb{Z}) .
$$


Then the sequence

$$
\cdots \rightarrow X_{k} \stackrel{T_{k}}{\rightarrow} X_{k+1} \rightarrow \cdots
$$

is called topologically exact.

TheOREM 3.6. Let $A, B$ and $C$ be Banach algebras where $\overline{A^{2}}=A$, and let $\phi: A \rightarrow B$ and $\psi: B \rightarrow C$ be continuous homomorphisms such that the sequence

$$
A \stackrel{中}{\rightarrow} B \stackrel{\Psi}{\rightarrow} C \rightarrow 0
$$

is exact. Then the sequence

$$
\Omega(A) \stackrel{\Omega(\phi)}{\longrightarrow} \Omega(B) \stackrel{\Omega(\psi)}{\longrightarrow} \Omega(C) \rightarrow 0
$$

is a topologically exact sequence of left Banach A-modules. Moreover, $\Omega(\psi)$ is onto.

Corollary 3.7. Let $A$ be a commutative Banach algebra, let $I \subset A$ be a closed ideal with $\bar{I}^{2}=I$, and let $\iota: I \rightarrow A$ be the natural embedding. Then the sequence

$$
\Omega(I) \stackrel{\Omega(\iota)}{\longrightarrow} \Omega(A) \stackrel{\Omega(\pi)}{\longrightarrow} \Omega(A / I) \rightarrow 0
$$

is topologically exact. Moreover, $\Omega(\pi)$ is onto.

Before we start proving Theorem 3.6, let us use Corollary 3.7 to obtain a hereditary property for weak amenability.

Corollary 3.8. Let $A$ be a commutative Banach algebra, and let $I \subset A$ be a closed ideal such that $I$ and $A / I$ are weakly amenable. Then $A$ itself is weakly amenable.

Proof. It remains to show that $\overline{I^{2}}=I$. To see this make $\mathbb{C}$ into a left Banach I-module by

$$
a \lambda=0 \quad(a \in I, \lambda \in \mathbb{C}) .
$$

Then any element of $I^{*}$ that vanishes on $\overline{I^{2}}$ is a continuous derivation. Hence, if $\overline{I^{2}} \neq I, I$ would not be weakly amenable.

This hereditary property can, of course, be obtained without involving the functor $\Omega$ (see [6]). Again, an analogous statement holds for amenable Banach algebras [8, Proposition 5.1]. It is a remarkable fact [5, Corollary 1.3] that a closed ideal in a weakly amenable Banach algebra is weakly amenable if and only if $\overline{I^{2}}=I$.

Proof of Theorem 3.6. First, we show that it is sufficient to prove Corollary 3.7. Put $I=\phi(A)$. Clearly, $I^{2}=I$ holds. Without loss of generality we may replace $C$ by $B / I$ and $\psi$ by $\pi: B \rightarrow B / I$, the canonical epinorphism. Let $\iota: I \rightarrow B$ denote the canonical embedding, and apply $\Omega$ to the complex

$$
A \stackrel{\oplus}{\rightarrow} I \stackrel{\leftrightarrow}{\rightarrow} B \stackrel{\pi}{\rightarrow} B / I \rightarrow 0 .
$$

We obtain:

$$
\Omega(A) \stackrel{\Omega(\phi)}{\longrightarrow} \Omega(I) \stackrel{\Omega(\iota)}{\longrightarrow} \Omega(B) \stackrel{\Omega(\pi)}{\longrightarrow} \Omega(B / I) \rightarrow 0 .
$$


By Proposition 3.4, $\Omega(\phi): \Omega(A) \rightarrow \Omega(I)$ is onto. Thus $\Omega(\phi) \Omega(A)$ is dense in $\operatorname{ker} \Omega(\pi)$ if and only if $\Omega(\iota) \Omega(I)$ is dense in $\operatorname{ker} \Omega(\pi)$. From Proposition 3.1, we already know that $\overline{\Omega(\iota) \Omega(I)} \subset \operatorname{ker} \Omega(\pi)$. By Lemma 3.3, $\Omega(B) / \operatorname{ker} \Omega(\pi)$ has a natural $B / I$-module structure. As we shall see in Lemma 3.9 , the same is true for $\Omega(B) / \overline{\Omega(\iota) \Omega(I)}$. There is a canonical continuous epimorphism $\rho: \Omega(B) / \overline{\Omega(\iota) \Omega(I)} \rightarrow \Omega(B) / \operatorname{ker} \Omega(\pi) \cong \Omega(B / I)$ of $B / I$-modules. If we can show that $\rho$ has a left inverse, we are finished. Define $\hat{d}_{B}: B / I \rightarrow \Omega(B) / \overline{\Omega(\iota) \Omega(I)}$ by

$$
\hat{d}_{B}(b+I)=d_{B} b+\overline{\Omega(c) \Omega(I)} \quad(b \in B) .
$$

Since $d_{B}(I) \subset \Omega(\iota) \Omega(I), \hat{d}_{B}$ is well defined. Clearly, $\hat{d}_{B}$ is a continuous derivation into a left Banach $B / I$-module. By Theorem 2.2 , there is a unique continuous $B / I$-module homomorphism $h: \Omega(B / I) \rightarrow \Omega(B) / \overline{\Omega(\iota) \Omega(I)}$ such that the diagram

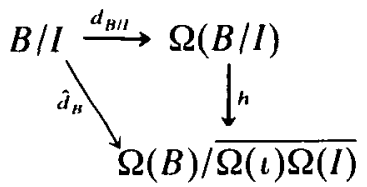

is commutative. Reasoning in the same way as in the proof of Proposition 3.4, it is not difficult to see that $h$ is the desired left inverse of $\rho$.

It remains to show that in the above proof $\Omega(B) / \overline{\Omega(\iota) \Omega(I)}$ has a $B / I$-module structure.

Lemma 3.9. Let $A$ be a commutative Banach algebra, let $I \subset A$ be a closed ideal with $\overline{I^{2}}=I$, and let $\iota: I \rightarrow A$ be the canonical embedding, then $\Omega(A) / \overline{\Omega(\iota) \Omega(I)}$ becomes a left Banach A/I-module via

$$
(a+I)(x+\overline{\Omega(\iota) \Omega(I)}):=a x+\overline{\Omega(\iota) \Omega(I)} \quad(a \in A, x \in \Omega(A)) .
$$

Proof. The first thing we have to show is that $\overline{\Omega(\iota) \Omega(I)}$ is an $A$-module. Since $\overline{I^{2}}=I, \Omega(\iota) \Omega(I)$ is generated by the elements $d_{A}(x y)$ with $x, y \in I$. For this reason, it is enough to show that given $a \in A$ and $x, y \in I$, the element $a d_{A}(x y)$ lies in $\Omega(\iota) \Omega(I)$. Since

$$
a d_{A}(x y)=a x d_{A} y+a y d_{A} x \quad(a \in A, x, y \in I),
$$

and $\overline{\Omega(\iota) \Omega(I)}$ is an $I$-module, this clearly holds. The rest of the proof is very similar to the proof of Lemma 3.3, so we omit it.

It is surely a flaw of Theorem 3.6 that given an exact sequence

$$
A \stackrel{\phi}{\rightarrow} B \stackrel{\psi}{\rightarrow} C \rightarrow 0,
$$

with $\overline{A^{2}}=A$ we cannot conclude the exactness of

$$
\Omega(A) \stackrel{\Omega(\phi)}{\longrightarrow} \Omega(B) \stackrel{\Omega(\psi)}{\longrightarrow} \Omega(C) \rightarrow 0 .
$$

The reason why our proof demonstrates merely the topological exactness of (6) is that we are concerned with Banach modules only. If we do not know whether $\Omega(\phi) \Omega(A)$ is closed, $\Omega(B) / \Omega(\phi) \Omega(A)$ need not be a Banach space. However, if we define the functor $\Omega$ in a purely algebraic context (as in [9]), one can show-adapting the proof of Theorem 3.6 -that this $\Omega$ preserves the exactness of (5) when $A^{2}=A$. 
4. Two fundamental topologically exact sequences. As far as algebraic differentials are concerned, two theorems referred to as the first and the second fundamental exact sequence are of particular importance [9, Theorem 57 and Theorem 58]. We want to prove analogues of these exact sequences in the Banach algebra context.

Before we can do so, we have to point out what we mean by a tensor product of left Banach modules over a commutative Banach algebra. We follow [7]. Let $A$ be a commutative Banach algebra, and let $M$ and $N$ be left Banach $A$-modules. Take the projective tensor product $M \otimes N$ of $M$ and $N$ considered as Banach spaces, and factor out the closed linear subspace generated by all tensors

$$
a x \otimes y-x \otimes a y \quad(a \in A, x \in M, y \in N) .
$$

We denote the resulting quotient space by $M \otimes_{A} N$ and the equivalence class of $x \otimes y$ by $x \otimes_{A} y$ for all $x \in M$ and $y \in N$. It causes little effort to verify that $M \otimes_{A} N$ has the universal property we want it to have: For each continuous $A$-bilinear mapping $\Psi: M \times N \rightarrow L$, where $L$ is another left Banach $A$-module, there is a continuous $A$-linear mapping $\Phi: M \otimes_{A} N \rightarrow L$ such that

$$
\Psi(x, y)=\Phi(x \otimes y) \quad(x \in M, y \in N) .
$$

We prove the functional analytic analogue of the second fundamental exact sequence first. Fix a commutative Banach algebra $A$, let $l \subset A$ be a closed modular ideal, and let $\pi: A \rightarrow A / I$ denote the canonical epimorphism. Define an $A / I$-linear continuous mapping $T: \Omega(A) \otimes_{A}(A / I) \rightarrow \Omega(A / I)$ by

$$
T\left(x \otimes_{A} a\right)=a \Omega(\pi) x \quad(x \in \Omega(A), a \in A / I) .
$$

Since $\Omega(\pi)$ is onto by Proposition 3.4 and $A / I$ is unital, $T$ is onto. Consider the map

$$
I \ni a \mapsto d_{A} a \otimes_{A} 1 \in \Omega(A) \otimes_{A}(A / I) .
$$

It vanishes on $\overline{I^{2}}$, and thus induces a continuous homomorphism of $A / I$-modules $S: I / \overline{I^{2}} \rightarrow \Omega(A) \otimes_{A}(A / I)$. Clearly, $S\left(I / \overline{I^{2}}\right) \subset \operatorname{ker} T$. We want to show that $S\left(I / \overline{I^{2}}\right)$ is dense in ker $T$. To see this, we regard the left Banach $A / I$-modules

$$
\mathrm{M}:=\left(\Omega(A) \otimes_{A}(A / I)\right) / \overline{S\left(I / \overline{I^{2}}\right)},
$$

and

$$
N:=\left(\Omega(A) \otimes_{A}(A / I)\right) / \operatorname{ker} T .
$$

There is a natural epimorphism $\rho: M \rightarrow N$. We show that $\rho$ has a left inverse. Define $D: A / I \rightarrow M$ by

$$
D(a+I)=d_{A} a \otimes_{A} 1+\overline{S\left(I / \overline{I^{2}}\right)} \quad(a \in A) .
$$

From the definition of $S$, this is a well defined, continuous derivation. Thus, there is a unique continuous homomorphism $h_{D}: \Omega(A / I) \rightarrow M$ such that $D=h_{D}{ }^{\circ} d_{A / l}$. On the other hand, $N$ and $\Omega(A / I)$ are topologically isomorphic by a mapping $\hat{T}$, which is canonically induced by $T$. It is not difficult to see that $h_{D} \circ \hat{T}^{-1}$ is a left inverse of $\rho$. Putting this all together, we have: 
THEOREM 4.1. Let $A$ be a commutative Banach algebra, and let $I \subset A$ be a closed modular ideal. Then

$$
I / \overline{I^{2}} \stackrel{S}{\rightarrow} \Omega(A) \bigotimes_{A}(A / I) \stackrel{T}{\rightarrow} \Omega(A / I) \rightarrow 0
$$

is a topologically exact sequence of left Banach A/I-modules. Moreover, $T$ is onto.

Before we turn to the first (topologically) exact sequence, we have to prove an extension of Theorem 2.2 .

Definition 4.2. Let $A$ and $B$ be commutative Banach algebras, let $\phi: A \rightarrow B$ be a continuous homomorphism, and let $M$ be a left Banach $B$-module. By a continuous $A$-derivation from $B$ into $M$ we mean a continuous derivation $D: B \rightarrow M$ such that $D \circ \phi=0$.

Theorem 4.3. Let $A$ and $B$ be commutative Banach algebras, and let $\phi: A \rightarrow B$ be a continuous homomorphism. Then there is a left Banach $B$-module $\Omega_{A}(B)$ and a continuous $A$-derivation $d_{B}^{A}: B \rightarrow \Omega_{A}(B)$ with the following universal property.

For each continuous $A$-derivation $D: B \rightarrow M$ into a Banach $B$-module $M$, there is a unique continuous homomorphism $h_{D}: \Omega_{A}(B) \rightarrow M$ of $B$-modules such that $D=h_{D} \circ d_{B}^{A}$. $\Omega_{A}(B)$ is unique up to topological isomorphism.

Proof. Take $\Omega(B)$, and factor out the closed submodule which is generated by $d_{B}(\phi(A))$. Define $\Omega_{A}(B)$ as the quotient module, and put $d_{B}^{A}=\pi_{A} \circ D_{B}$, where $\pi_{A}: \Omega(B) \rightarrow \Omega_{A}(B)$ is the canonical epimorphism. It is easy to see that $\Omega_{A}(B)$ and $d_{B}^{A}$ have the desired universal property.

If $A$ is a commutative Banach algebra, and $\phi: \mathbb{C} \rightarrow A$ is an arbitrary homomorphism, then $\phi(\mathbb{C})$ consists of the scalar multiples of the idempotent $\phi(1)$. Therefore, continuous $\mathbb{C}$-derivations are just derivations in the usual sense, and we have $\Omega_{\mathbb{C}}(A)=\Omega(A)$ and $d_{A}^{\complement}=d_{A}$.

Now, fix two commutative Banach algebras $A$ and $B$, assume $B$ to have an identity element 1 , and let $\phi: A \rightarrow B$ be a continuous homomorphism. Let $R: \Omega(B) \rightarrow \Omega_{A}(B)$ denote the map $\pi_{A}$ from the proof of Theorem 4.3, and define $T: \Omega(A) \otimes_{A} B \rightarrow \Omega(B)$ by

$$
T\left(x \otimes_{A} b\right)=b \Omega(\phi) x \quad(x \in \Omega(A), b \in B) .
$$

Define

$$
M:=\Omega(B) / \overline{T\left(\Omega(A) \hat{\otimes}_{A} B\right)},
$$

and let $\pi: \Omega(B) \rightarrow M$ be the canonical epimorphism. Since $T\left(\Omega(A) \otimes_{A} B\right)$ is clearly contained in the kernel of $R$, there is a natural epimorphism $\rho: M \rightarrow \Omega_{A}(B)$. Further, since

$$
T\left(d_{A} a \otimes_{A} 1\right)\left(d_{B}^{\circ} \phi\right) a \quad(a \in A),
$$

$D=\pi \circ d_{B}$ is a continuous $A$-derivation from $B$ into $M$. Hence, there is a unique continuous homomorphism $h_{D}: \Omega(B) \rightarrow M$ such that $D=h_{D} \circ d_{B}^{A}$; it is easily seen to be a left inverse of $\rho$. 
Theorem 4.4. Let $A$ and $B$ be commutative Banach algebras, let $B$ be unital, and let $\phi: A \rightarrow B$ be a continuous homomorphism. Then

$$
\Omega(A) \hat{\otimes}_{A} B \stackrel{T}{\rightarrow} \Omega(B) \stackrel{R}{\rightarrow} \Omega_{A}(B) \rightarrow 0
$$

is a topologically exact sequence of left Banach B-modules. Moreover, $R$ is onto

\section{REFERENCES}

1. W. G. Bade, P. C. Curtis and H. G. Dales, Amenability and weak amenability for Beurling and Lipschitz algebras, Proc. London Math. Soc. (3) 55 (1987), 359-377.

2. F. F. Bonsall and J. Duncan, Complete normed algebras (Springer Verlag, 1973).

3. P. C. Curtis and R. J. Loy, The structure of amenable Banach algebras, J. London Math. Soc. (2) 40 (1989), 89-104.

4. H. Grauert and R. Remmert, Analytische Stellenalgebren (Springer Verlag, 1971).

5. N. Grønbæk, Commutative Banach algebras, module derivations, and semigroups, $J$. London Math. Soc. (2) 40 (1989), 137-157.

6. N. Grønbæk, A characterization of weakly amenable Banach algebras Studia Math. XCIV (1989), 149-162.

7. A. Ya. Helemskii, The homology of Banach and topological algebras, (Kluwer Academic Publishers, Dordrecht-Boston-London, 1989).

8. B. E. Johnson, Cohomology in Banach algebras, Memoirs of the AMS 127 (1972).

9. H. Matsumura, Commutative algebra (W. A. Benjamin, New York, 1970).

Department of Mathematics

UNIVERSITY OF CALIFORNIA

BERKELEY, CA 94720

USA 